\title{
Design and Development of Mobile Learning System Based on
}

\section{Internet}

\author{
Zhengru Xu, Weili Chu,Yanmei Meng
}

\author{
Qingdao Huanghai University, Qingdao, Shandong, China
}

\begin{abstract}
In this paper, it takes the theory of education and educational technology as the foundation, which mainly takes the construction of mobile learning system theory and method as the main research contents, meanwhile it can realize the semantic query function, so as to provide methodological enlightenment for designing mobile learning system, which also can provide technical support for the personalized mobile context personalized design.
\end{abstract}

Keyword: Semantic Web, Mobile learning system, Internet, E-learning

\section{Introduction}

Semantic Web was formally proposed by Berners-Lee in 1999, who was also the founder of W3C. Educators had great interest in the emergence of educational semantic web. It is the research focus of Semantic Web that the semantic meaning can be understood and processed by computer, which can expand the current network information. While facilitating the interaction between human and computer is the purpose of Semantic Web. [1] At present, more and more researches on the learning domain of semantic web are presented, such as the realization of learning content database based on Semantic Web, as well as the research on the semantic retrieval of teaching management system based on Semantic Web.The realization of learning content database based on Semantic Web can only construct the learning database, which can not involve the retrieval method, while the research on the semantic retrieval of teaching management system based on Semantic Web only puts its focus on the study of paper retrieval. This paper mainly focuses on the application and implementation of semantic query method in learning content.

Mobile learning has been born as a new form of learning as well as a new type of learning technology, the design and development of mobile learning system is the basis for the development and implementation of mobile learning. This paper studies the theory and method of constructing mobile learning system, which aims at providing methodological enlightenment for the design and development of mobile learning system, so as to use the design idea of semantic retrieval, and explore the design method of semantic retrieval in mobile learning system. [2] In the whole designing process, the system will be based on the learner's style characteristics, learning needs, abilities, level, environment as well as the other factors, to provide them with the corresponding learning content and learning strategies of fast links. At the same time, this paper can realize the semantic retrieval in the learning content, so that learners can find out what they need to learn according to their own needs, which can ultimately improve learning initiative and enthusiasm of learners. 


\section{Architecture of Semantic Web}

The future of Internet development is semantic web, simply speaking , semantic web can be divided into two parts, namely, "semantics" and "Internet". As for semantics, it refers to the relationship between the objects, while Internet can show that semantics is an extension of the existing network, semantics can help the existed network to play its advantages, rather than to create a new network. In the early developing days of semantic web, people put too much emphasis on the meaning of semantics, [3] but now people put more emphasis on network. Therefore, firstly semantic web should be network then semantics, and semantics is only a kind of emphasis on the meaning, not the formal semantic in the true meaning. At the same time, the designing process of semantic web should follow certain principles: all resources should be identified with URI; [4] resource and connection can be allowed to have many types; part, fragment and incomplete information can be allowed to exist; information need not be absolutely true; support and reflect the change and evolution of information; minimum designing principle. According to these designing principles, the semantic web hierarchy was proposed by TimBerners-Lee in 2000, which can be shown in Fig.1.

\section{(1) Unicode and URI Layer}

Unicode and URI can be acted as the bottom of seven layer, Unicode can process the codes of resources, URI is the superset of the uniform resource location method. The former can ensure that the international character set is used, so as to realize the unified coding of online information, while the latter can support and make it possible that the identified accurate information of semantic web objects and resources can be retrieved.

\section{(2) XML+NameSpace+XMLSchema}

This layer includes name space and definition of XMLSchema, which can make people use their own definition of vocabulary to create structured document and make communication on the Internet.

\section{(3) RDF+RDFSchema Layer}

RDF is the basic data model of semantic web, which can be used to describe the network resources. RDF data model does not depend on XML, but it follows the syntax of XML to express the complex relationship between online objects.

\section{(4) Ontology Layer}

This layer is used to describe the connection between various resources, adopting OWL to express. Separating the structure and content of information, then it can make a formal description, and computer can understand its meaning.

\section{(5) Logic Layer}

This layer is mainly the production of rules, which can provide the basis for reasoning mechanism, it can provide rules as well as reasoning rules for logical subjects. 


\section{(6) Proof Layer}

This layer combines the application mechanism of the trust level to judge the rules generated by the upper layer. This layer is highlighted and emphasized on the provision of authentication mechanisms.

\section{(7) Trust Level}

This layer is to deepen the function of the upper layer, so as to ensure the reliability of personalized service and cooperation, this layer puts more emphasis on the emphasis of the trust mechanism.

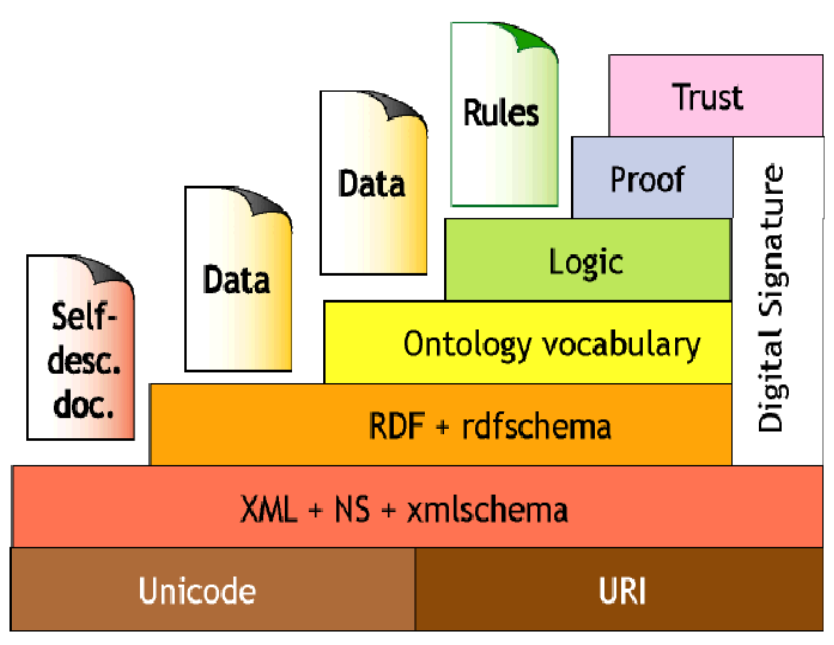

Fig.1 Architecture of Semantic Web

Mobile learning is the practical application of mobile technology, computer network as well as communication technology to education, which is a kind of extension and expansion of digital learning. In this paper, the learning environment can be divided into classroom learning environment, E-learning learning environment and mobile learning environment. Then, based on the disadvantages and advantages of these three kinds of learning environments, it can provide guidance for the construction of mobile learning environment. Distance learning can realize the separation of teaching and learning during the process of learning, paying attention to the flexibility of learning, so as to pursue the organizational level of learning. Digital learning can solve learning problems in the digital world, focusing on the technical problems of learning. Mobile learning is the closest situation stage of their development, which can guide learners to learn effectively by using communication technology and information technology through learning technology. In this process, we should pay more attention to the differences of learners, as well as the development and design of learning system, put more focus on learners' learning". Table 1 is the comparison of characteristics of these three learning environments. 
Table 1 Comparison of Characteristics of Three Learning Environments

\begin{tabular}{|c|c|c|c|}
\hline Comparison items & $\begin{array}{c}\text { Learning environment } \\
\text { in traditional }\end{array}$ & E-learning environment & $\begin{array}{c}\text { Mobile learning } \\
\text { environment }\end{array}$ \\
\hline Characteristics of learning & Learning is constrained & $\begin{array}{l}\text { Learning is without } \\
\text { restraint }\end{array}$ & $\begin{array}{l}\text { Using a short period of } \\
\text { time to carry out }\end{array}$ \\
\hline Teaching design & $\begin{array}{c}\text { Learning objective } \\
\text { oriented }\end{array}$ & Learner oriented & Activity oriented \\
\hline Teacher's role & Leading position & Facilitator & Community of learning \\
\hline The used technical means & Teaching materials & Fixed computer & Mobile terminal \\
\hline Limitation of place and time & $\begin{array}{c}\text { Fixed location; Fixed } \\
\text { time }\end{array}$ & Time without limitation & $\begin{array}{l}\text { Time without limitation; } \\
\text { Place without limitation }\end{array}$ \\
\hline
\end{tabular}

\section{Functional Structure Model of Mobile Learning System Based on Semantic Web}

Under the environment of mobile learning, in order to make learners obtain key learning content timely, so as to make teachers or experts guide learners timely, the appropriate solution to learning content as well as information structure model related to learning should be solved correspondingly. As for mobile learning, it is key to pay attention to intelligent adaptive transmission and situation learning. As for learners, they need access to information by using their fragmented time, which is the ultimate goal of modern learning, at the same time, knowledge acquisition and application of knowledge can be effectively combined, which is the essence of learning.

The main functional module of this mobile learning system includes: (1) Learners module, the design and implementation of this module is usually carried out by relational database in the program, in the mobile learning system, learners are the core of this functional module. (2) Learning content modules, which are generally presented in the structured form or in the form of documents, a lot of information and knowledge that learners needed can be stored in this module, the structured learning content is usually based on XML to describe the knowledge point, the access to the form of document is from memory to read and call. (3) Interaction module, which is also the module that users interact with the machine, in the mobile learning system, the interaction module does not have the unified standard, but it pays more attention to the situation consciousness, so as to adapt to the needs of the learners timely. (4) Situation consciousness module, which is an important functional module in mobile learning system. In mobile learning system, the module can match the corresponding learning content according to the characteristics of learners, learning content as well as the static characteristics of learning goals, by using intelligent algorithms and techniques to realize the module.

\section{Conclusion}

In this paper, the reference model of mobile learning technology system is discussed in detail from the perspective of learning technology system, on the basis of mobile technology 
system as well as mobile learning application system, it can build the functional structure model of mobile learning based on Semantic Web.

\section{Reference}

[1] Chen, Yuh-Shyan, Tai-Chien Kao, and Jay-Ping Sheu. "A mobile learning system for scaffolding bird watching learning." Journal of Computer Assisted Learning 19.3 (2003): 347-359.

[2] Tan, Tan-Hsu, and Tsung-Yu Liu. "The mobile-based interactive learning environment (MOBILE) and a case study for assisting elementary school English learning." Advanced Learning Technologies, 2004. Proceedings. IEEE International Conference on. IEEE, 2004.

[3] Jin Y. Research of one mobile learning system[C]//Wireless Networks and Information Systems, 2009. WNIS'09. International Conference on. IEEE, 2009: 162-165.

[4] He, Shuai. "Lean Six Sigma Implementation in Chinese Manufacturing SMEs." Journal of Applied Science and Engineering Innovation 3.3 (2016): 105-112. 